\title{
Semantic Similarity Models for Geo-spatial Information System
}

\author{
K.Saruladha, PhD. \\ Department of Computer Science and Engineering, \\ Pondicherry Engineering College, \\ Puducherry, India.
}

\author{
E.Thirumagal \\ Department of Computer Science and Engineering, \\ Pondicherry Engineering College, \\ Puducherry, India.
}

\begin{abstract}
Semantic similarity measurement is the practice of estimating the relatedness of the concept based on the likeness of their meaning or their semantic content. Today's increasing interest on the geospatial information system, leads to development of the query system which needs to provide efficient geo-spatial information retrieval. The properties and spatial relations between the geo-spatial concepts must be taken into account for retrieving geo-spatial information efficiently. This paper provides the survey on various models such as geometric model, network model, transformation model, hybrid model, etc, for discovering the semantic similarity between the geo-spatial concepts. It also focuses the drawbacks of each semantic similarity model and depicts how the hybrid model works well when compared with other models in retrieving the Geo-spatial information efficiently. The main objective of this paper is to propose Hybrid semantic similarity model using Manhattan distance and by considering contexts of the Geo-spatial concepts. The Manhattan distance method is used to estimate the semantic distance between the Geo-spatial concepts and to retrieve the Geo-spatial information efficiently. The position of the locations is identified by using the map similarity.
\end{abstract}

\section{General Terms}

Artificial Intelligence, Semantic Web, Knowledge sharing, Knowledge Representation.

\section{Keywords}

Geo-spatial information retrieval, semantic similarity, hybrid model, Manhattan distance, computational models, spatial relations.

\section{INTRODUCTION}

Geospatial information system plays a significant role to help people to collect and analyze the related spatial data. Geospatial information system has its application in various areas in that, most significant areas are natural resources management (which includes management of habitat of wildlife, management of floodplains, wetlands and agricultural lands), facilities management (which includes management of electrical network load balancing and locating underground pipes), land management(includes management of land acquisition, water quality management, etc). For the better design of the geographical information system, it is important to afford adequate knowledge representations and the similarity measures. Geospatial ontology [1] plays a vital role in providing the semantics of the geo-spatial concepts and also used for identifying the semantically similar concepts. The properties and spatial relations [2] between the geo-spatial concepts must be taken into account for retrieving geo-spatial information efficiently. The Geo-spatial ontology [3] for Hydrological concepts and its relations are shown in Fig.1.The properties are the features or the characteristics that describe the geo-spatial concept. The geographic feature of the one is related to the other geographic feature of the geo-spatial concept specifies the spatial relation [4]. The Geospatial relations and its descriptions are shown in Table 1.The knowledge representations were first used in the psychology [5] and the concepts are modelled within the multidimensional space. All geospatial objects are located in N-dimensional space.

There are four steps involved in the semantic information retrieval task such as

- The semantic description of the data source provided by some ontology.

- The semantic description of the query extracted from the specified ontology.

- The query concept is enriched by matching it with the data source concepts using semantic similarity measures to improve the information retrieval.

- The results (information retrieved) are returned to the user.

The notion of similarity includes both the commonalities and the differences. When commonality between the geo-spatial concepts is more, it indicates two concepts are very similar. In the Geo-spatial domain, the semantic distance may be spatial distance or the path distance. This paper focuses the survey on various models for estimating the semantic similarity among the geo-spatial concepts. The various models of similarity such as Geometric model [6], Alignment model [7], Feature model [8], Network model [9], Transformational model [10], could be classified as shown in the Fig 2. This classification is based on how concept properties and concept relations quantify similarity among the Geo-spatial concepts.

To overcome the difficulties of the above said model, the new model emerged known as Hybrid model [2], which is the combination of the geometric and the network models for specifying both the properties and the spatial relations [11] of the geospatial concept. This paper reports the drawbacks of the various semantic similarity models and depicts how the hybrid model works well when compared to other models. In Hybrid model, Euclidean distance method [2] is used for manipulating the semantic distance. 


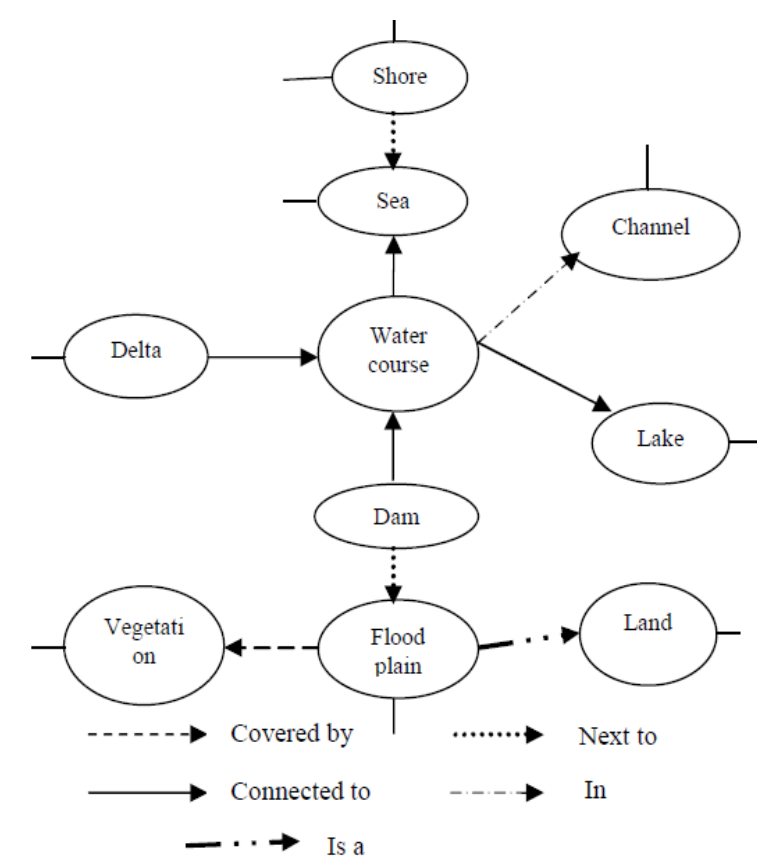

Fig 1: Geo-spatial ontology for Hydrological concepts

In this paper, the proposed Hybrid Model which is the combination of the Geometric and Network model is different from existing models in two aspects.

- Instead of Euclidean distance method, Manhattan distance method [12] for manipulating the semantic distance for easier and efficient computation.

- Further the contexts are identified by assigning weights to the Geo-spatial concepts in the ontology and the position of the locations [13] is identified by using the map based similarity.

Table 1. Geo-Spatial Relations and its Description

\begin{tabular}{|l|l|}
\hline $\begin{array}{l}\text { Geospatial } \\
\text { Relations }\end{array}$ & \multicolumn{1}{|c|}{ Description } \\
\hline Is a & $\begin{array}{l}\text { The child concept inherits instances from the } \\
\text { parent concept. }\end{array}$ \\
\hline Next to & $\begin{array}{l}\text { All the instances are related to at least one } \\
\text { instance of the other concept }\end{array}$ \\
\hline $\begin{array}{l}\text { Connected } \\
\text { to }\end{array}$ & $\begin{array}{l}\text { All the instances of each concept have spatial } \\
\text { relations to each other. }\end{array}$ \\
\hline in & $\begin{array}{l}\text { All the instances of one concept are present in } \\
\text { another concept. }\end{array}$ \\
\hline Covered by & $\begin{array}{l}\text { All the instances of each concept have spatial } \\
\text { relations to each other over the period of time. }\end{array}$ \\
\hline
\end{tabular}

In section 2, the classification of the computational models based on the properties, relations and transformations and its limitations are discussed. In section 3, the comparison of the various computational models is made. In section 4 , the Proposed Hybrid Model is depicted. In section 5, the conclusions derived and the future enhancements are mentioned.

\section{CLASSIFICATION OF SEMANTIC SIMILARITY COMPUTATIONAL MODELS}

There are various computational models for finding semantic similarity among geospatial concepts. The computational models are classified based on the concept properties and concept relations as shown in Fig.2. As said earlier the properties are the characteristics that describe the geo-spatial concept. The geographic feature of one Geo-spatial concept is related to the geographic feature of another Geo-spatial concept specifies the spatial relation. In transformation model, the similarity is quantified based on the number of changes made to translate from one geo-spatial concept to another geospatial concept.

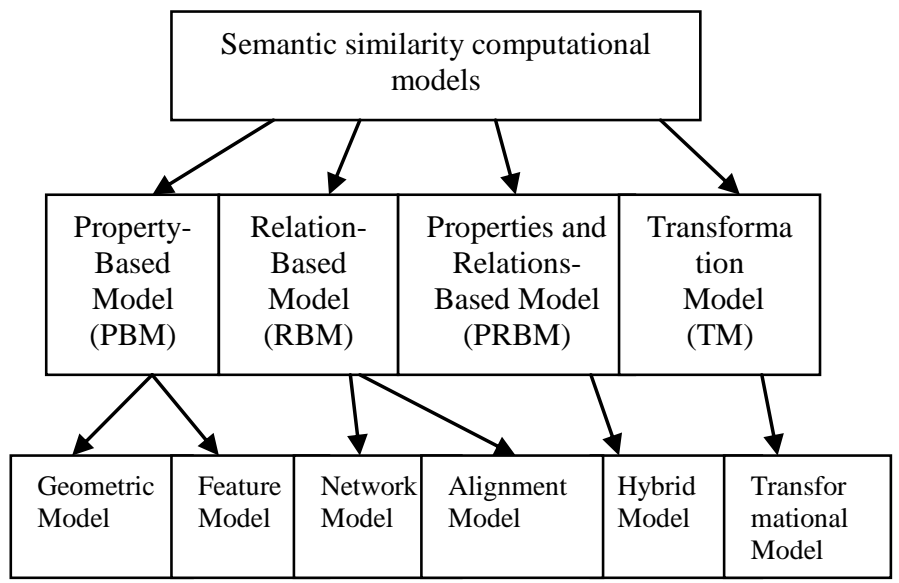

Fig 2: Classification of semantic similarity computational models

\subsection{Property-Based model (PBM)}

Properties are the characteristics that describe the geospatial concept. For example the geo-spatial concept "Floodplain" has the properties of flat gradient, waterlogged, inhabited, land surface, etc. The semantic similarity models that concentrate only on properties are Geometric model and Feature model. These models do not consider the spatial relations between the geo-spatial concepts which lead to ineffective information retrieval. PBM includes Geometric model and Feature model discussed in next two below sections.

\subsubsection{Geometric model}

The geometric model of representation was first used in the psychology and here the concepts are modelled within the Ndimensional space [Multidimensional Scaling (MDS)] and the spatial distance between the concepts quantify the semantic similarity between the geospatial concepts. MDS [6] is the mathematical model of Categorization, identification, recognition, memory and generalization. The input of MDS is set of stimuli. Stimuli are represented by points arranged in space according to their pair wise distances (i.e.) maximum distance $\mathrm{d}-1$ used to arrange $\mathrm{d}$ stimulus. The spatial representation of geometric similarity is given by the matrix below.

Quality1 Quality2......... Quality

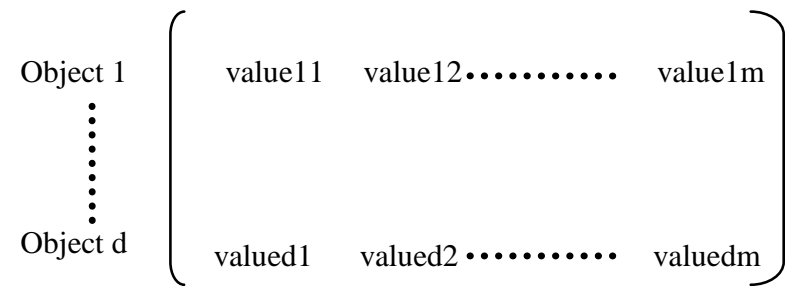


Quality - dimension of the space (properties).

Values - properties described via value on the quality dimension.

The various objects here are compared with the various dimensions using the values. The Minkowski distance measure $\mathrm{d}_{\mathrm{ij}}$ is given by equation (1) is used for measuring the semantic similarity among geo-spatial concepts.

$$
\mathrm{d}_{\mathrm{ij}}=\left(\sum_{\mathrm{k}=1}^{\mathrm{n}}\left|\mathrm{x}_{\mathrm{ik}}-\mathrm{x}_{\mathrm{jk}}\right|^{\mathrm{r}}\right)^{1 / \mathrm{r}}
$$

$\mathrm{n}$ - Number of dimensions. $\mathrm{x}_{\mathrm{ik}}$ is the dimensional value of dimension $\mathrm{k}$ for the stimulus $\mathrm{i} . \mathrm{x}_{\mathrm{jk}}$ is the dimensional value of dimension $\mathrm{k}$ for the stimulus $\mathrm{j}$. when $\mathrm{r}=1$, city block distance method is used. When $\mathrm{r}=2$, Euclidean distance method is used. In the next subsection, Feature model is described.

\subsubsection{Feature Model}

As such the Geometric model, in Feature model the geospatial concepts are described using its properties. In the feature model, the features of the Query Q and the Concept C in the data source are matched which uses the Boolean values. The representation model of the feature model [14] consists of the bag of the features for each concept. For example the concept wetland consists of the features such as flat, low vegetation, often water logged, etc.

The Query Q contains the set of features. The Concept C in data source consists of set of features. The matching features of the $\mathrm{Q}$ and $\mathrm{C}$ are given by $\mathrm{Q} \cap \mathrm{C}$. The feature model uses the Matching Distance Similarity Measure (MDSM) [2] for measuring the semantic distance between the geo-spatial concepts. Here the concepts are in the tree ontology. The semantic similarity distance is obtained by measuring matching depth distance among two concepts. The semantic distance $\mathrm{S}(\mathrm{Q}, \mathrm{C})$ among the geo-spatial query concept $\mathrm{Q}$ and the related geo-spatial concept $\mathrm{C}$ in the data source is given by equation (2).

$S(Q, C)=\frac{f(Q \cap C)}{f(Q \cap C)+\alpha * f(Q-C)+\beta * f(C-Q)}$

where,

$$
\alpha=\left\{\begin{array}{l}
\frac{\operatorname{depth}(Q)}{\operatorname{depth}(Q)+\operatorname{depth}(C)}, \operatorname{depth}(Q) \leq \operatorname{depth}(C) \\
1-\frac{\operatorname{depth}(Q)}{\operatorname{depth}(Q)+\operatorname{depth}(C)}, \operatorname{depth}(Q)>\operatorname{depth}(C)
\end{array}\right.
$$

$$
\beta=1-\alpha
$$

where $\alpha$ and $\beta$ are the ratio of the Geo-spatial query and Geospatial concept in data source depths in the ontology described by Rodriguez and Egenhofer [14]. In the above two models, only the properties are considered and relations are ignored while quantifying the similarity among the Geospatial concepts. The Relation-Based Model is discussed in next section.

\subsection{Relation-Based Model (RBM)}

The geographic feature of the one is related to the other geographic feature of the geo-spatial concept specifies the spatial relation. In the Property-Based Model (PBM), the properties are used to describe the concepts; here the relations are used to describe the concepts. Some of the Geo-spatial relations between the Geo-spatial concepts are next to, nearby, going to, coming from, adjacent to, connected to, contained within, covered by, towards, is a, part of, run along, run into, etc. The RBM includes Network model and Alignment model discussed in next two below sections.

\subsubsection{Network model}

The network model [15] uses the graph-theory and it uses the semantic networks for the knowledge representation. Its representational model consists of the orientation indicates the connection between the two geo-spatial concepts (unidirectional, bidirectional and undirected), the labels i.e. the relation between the two concepts (is a, part of, located) and the weighting i.e. how they are connected (lightly connected, moderately connected, strongly connected). Here the trees of the concepts which are related to one another are made and whose probability increases in the upward direction where the subset of nodes is connected to the parent node in the bottom up approach. The semantic similarity distance is given by,

$$
\operatorname{Dis}(\mathrm{q}, \mathrm{c})=\operatorname{minlen}\left(\mathrm{P}_{\mathrm{q}}, \mathrm{c}\right)
$$

In equation (3), Dis(q, c) is the semantic similarity distance among the geo-spatial query concept and the related geospatial concept in the data source. $\left(\mathrm{P}_{\mathrm{q}}, \mathrm{c}\right)$ is the path length of every matching combinations of nodes in the network. The semantic neighbourhood [9] is made in the network model. The relation among the geo-spatial concepts is the potency of the network model. In next section the Alignment model is discussed.

\subsubsection{Alignment model}

This model estimates the alignment between the relational structures for the semantic similarity measurement. The PBM explores only the matching elements, but the alignment model will estimate whether the matching elements are aligned or not. Consider two concepts such as "I am watching my sister" and "my sister is watching television" then the alignment can be made in the way that "I am watching my sister watching television". Goldstone [4] introduced Similarity, Interactive Activation, and Mapping (SIAM) model for discovering the semantic similarity distance among the geo-spatial concepts is the alignment model. This SIAM involves various steps for finding the semantic similarity such as, aligning the features between the concepts, aligning the components between the concepts, component and the feature nodes are connected by the relations, on comparing the feature values of alignable feature slots, the match values are computed. The process of finding semantic similarity using the transformations is described in next section. 


\subsection{Transformation Model(TM)}

The transformation is that number of changes made to transform one geo-spatial concept to another geo-spatial concept. The transformational model uses the process of transformation to find the semantic similarity between the geo-spatial concepts which is discussed in next section.

\subsubsection{Transformational model}

The transformational model is that the number of changes or transformations that are made to transform one concept to another similar concept. The semantic similarity distance among the Geo-spatial concepts is the number of transformations or changes that are made. When the numbers of transformations are less, then similarity is more. When the numbers of transformations are more, then the similarity is less. Levenshtein edit distance [16] is the most famous transformational semantic similarity measure uses three transformations namely deleting, inserting and replacing letters in the word.

\begin{tabular}{|l|l|} 
1. replace(c,m) watercourse & watercourse \\
2. replace(o,e) watermeurse & 1.replace(o,h) waterchurse \\
3.replace(u,a) watermearse & 2.replace(u,a) watercharse \\
4.replace(r,d) watermeadse & 3.replace(r,n) waterchanse \\
5.replace(s,o) watermeadoe & 4.replace(s,n) waterchanne \\
6.replace(e,w) watermeadow & 5.insert(l,e) waterchannel \\
watermeadow & waterchannel \\
\hline
\end{tabular}

Fig 3: Transformation of the concept Water course to its related concepts.

From Fig 3, the water meadow has 6 transformations and the water channel has 7 transformations, so the semantic similarity is more for watermeadow than water channel. The next section discusses about the comparison of the existing semantic similarity computational models.

\subsection{Properties and Relations-Based Model (PRBM)}

The properties and the relations are used for describing the geo-spatial concepts which is the most effective way of retrieving the similar geo-spatial data. The hybrid model uses both the dimensions of the concept and the relations between the various concepts. The existing hybrid model is described in next section.

\subsubsection{Existing Hybrid model}

The hybrid model [2] is the combination of the geometric and the feature model. This model enables the concept to be described using the dimensions of the concept as well as the relations of the concept. In the hybrid model the $\mathrm{N}$ dimensional problem is reduced to one dimensional problem. In this Hybrid model the Euclidean distance method is used for finding the semantic similarity between the Geo-spatial concepts which leads to more number of computational steps.
The limitations of existing computational models are described in next section.

\subsection{Limitations of Existing computational models}

Geometric Model. In Geometric model it is difficult to specify the scope of the domain or dimensions in the conceptual space and the similarity judgement cannot be made only the semantic distance alone calculated.

Feature Model. Because of the large possible compound features (features match or not) will not lead to good measurements of the semantic similarity in case of the Feature model.

Network Model. In the network model, only the spatial relations between the concepts are described and the properties are not described. The process of finding semantic neighbourhood is an extra step which is not needed.

Alignment Model. In the Alignment model, there are possibilities for the two concepts can maps to the single concept which is inconsistent.

Transformational Model. This model is not suitable for complex tasks. It reflects the same drawback of the geometric model that it can estimate only the semantic distance.

Existing Hybrid Model. The existing Hybrid model uses the Euclidean distance method to calculate the semantic distance which is computationally difficult and does not produce better precision and recall. The next section describes the comparison of various existing computational models.

\section{COMPARISON OF THE EXISTING SEMANTIC SIMILARITY COMPUTATIONAL MODELS}

In this section the above computational models are compared. For the query "flooding area" given by the user, the most similar data source concept is "floodplain" for which the precision and recall values calculated for each model is shown in Table 2. This section also describes the drawbacks of the various computational models of semantic similarity measurement between the Geo-spatial concepts and depicts how well the Hybrid model works. We can infer that the hybrid model provides the reasonable precision and the recall when compared to other models of similarity from the Table.2. For comparison of various Geo-spatial semantic similarity models, three data sources are used namely

- OS (Ordnance Survey) Master map which is the national geographic database of Great Britain uses 28 Geo-spatial concepts and 15 distinct spatial relations among those concepts.

- ATKIS (Amtliches Topographisch Kartographisches Information System) which is the official topographic information system of Germany uses 18 Geo-spatial concepts and 9 distinct spatial relations among them.

- $\quad$ SDTS (Spatial Data Transfer Standard) which is the American national standard for information exchange uses 23 Geo-spatial concepts and 13 distinct spatial relations among those concepts. 
Table 2. Comparison of existing semantic similarity computational models for the geo-spatial Concept "Floodplain"

\begin{tabular}{|c|c|c|c|c|}
\hline $\begin{array}{l}\text { Semantic Similarity } \\
\text { computational model }\end{array}$ & Datasource & Precision & Recall & Drawbacks \\
\hline \multirow[t]{4}{*}{ Geometric model } & OS MasterMap ${ }^{\circledR}$ & & & \multirow{4}{*}{$\begin{array}{l}\text { It is difficult to specify the scope of the domain } \\
\text { or dimensions in the conceptual space and the } \\
\text { similarity judgement cannot be made only the } \\
\text { semantic distance alone calculated which } \\
\text { ignores the Geo-spatial relations. }\end{array}$} \\
\hline & & 1.00 & 0.17 & \\
\hline & ATKIS & 1.00 & 0.25 & \\
\hline & SDTS & 0.40 & 0.00 & \\
\hline \multirow{5}{*}{ Feature model } & OS MasterMap® & & & \multirow{5}{*}{$\begin{array}{l}\text { Because of the large possible compound } \\
\text { features(features match or not) will not lead to } \\
\text { good measurements of the semantic similarity }\end{array}$} \\
\hline & & 0.80 & 0.11 & \\
\hline & ATKIS & & & \\
\hline & & 0.60 & 0.15 & \\
\hline & SDTS & 0.80 & 0.16 & \\
\hline \multirow{4}{*}{ Network model } & OS MasterMap® & & & \multirow{4}{*}{$\begin{array}{l}\text { Only the relations between the concepts are } \\
\text { described and the properties are not described. } \\
\text { The process of finding semantic neighbourhood } \\
\text { is an extra step which is not needed. }\end{array}$} \\
\hline & & 0.42 & 0.00 & \\
\hline & ATKIS & 0.60 & 0.15 & \\
\hline & SDTS & 0.19 & 0.00 & \\
\hline \multirow{4}{*}{ Alignment model } & OS MasterMap® & & & \multirow{4}{*}{$\begin{array}{l}\text { Here there is a possibility for the two concepts } \\
\text { can maps to the single concept which is } \\
\text { inconsistent. This model is not suitable for } \\
\text { complex tasks. }\end{array}$} \\
\hline & & 1.00 & 0.17 & \\
\hline & ATKIS & 1.00 & 0.25 & \\
\hline & SDTS & 0.40 & 0.00 & \\
\hline \multirow[t]{3}{*}{$\begin{array}{l}\text { Transformational } \\
\text { model }\end{array}$} & OS MasterMap® & 0.80 & 0.41 & \multirow[t]{3}{*}{$\begin{array}{l}\text { Same as that of the geometric model, it can } \\
\text { estimate only the semantic distance. }\end{array}$} \\
\hline & ATKIS & 0.60 & 0.30 & \\
\hline & SDTS & 0.80 & 0.35 & \\
\hline \multirow{3}{*}{$\begin{array}{l}\text { Existing Hybrid } \\
\text { Model }\end{array}$} & OS MasterMap ${ }^{\circledR}$ & 0.80 & 0.69 & \multirow{3}{*}{$\begin{array}{l}\text { Computation is difficult and does not provide } \\
\text { best precision and recall. }\end{array}$} \\
\hline & ATKIS & 1.00 & 0.29 & \\
\hline & SDTS & 0.87 & 0.68 & \\
\hline
\end{tabular}




\section{PROPOSED HYBRID MODEL}

For the Query given by the user and the related geo-spatial concept in datasource, all the dimensions are obtained. All the matching and non-matching dimensions of the Query given by the user and geo-spatial concept in data source are obtained. When using the Euclidean distance method in Hybrid Model, the computation is difficult and does not produce best precision and recall values. The Manhattan distance method when used for Image Retrieval Application has yielded better precision. The Manhattan distance method is not used for computing semantic similarity in the geo-spatial domain. So the Manhattan distance [17] is proposed for computing semantic similarity between the Geo-spatial concepts. The architecture of the proposed Hybrid Model is shown in the Fig 4. The general Manhattan distance is computed using equation (4) is the easiest and efficient computation method and provides good precision and recall

Manhat tan distance $=\left|a_{1}-b_{1}\right|+\left|a_{2}-b_{2}\right|+\cdots+\left|a_{n}-b_{n}\right|$

In the general Manhattan distance [12] given by equation (4), $a_{1}, a_{2}, \ldots . . a_{n}$ and $b_{1}, b_{2}, \ldots . b_{n}$ are the points in $\mathrm{N}$ dimensional space. The points in the general Manhattan distance equation are mapped as the dimensions or properties of the geo-spatial concept in $\mathrm{N}$-dimensional space in the geospatial domain. The general Manhattan distance is mapped to geo-spatial domain which is given by equations (5), (6) and (7). Manhattan distance is calculated for the dimensions and the relations of the Query concept and the concept in the data source. The dimensional semantic distance semdist (dim) is calculated using equation (5), where $D_{\text {dimstd }}(\operatorname{dimq})$ is the standard dimensional distance of the query and $\mathrm{D}_{\text {dimman }}(\mathrm{Q}, \mathrm{C})$ is the Manhattan distance between the query and the concept in the data source

Semdist $(\operatorname{dim})=\alpha^{*} \mathrm{D}_{\text {dim std }}(\operatorname{dimq})+\beta^{*} \mathrm{D}_{\operatorname{dim} \operatorname{man}}(\mathrm{Q}, \mathrm{C})$

The relational semantic distance semdist (rel) is calculated using equation (6), where $D_{\text {relstd }}($ relq) is the standard relational distance of the query and $\mathrm{D}_{\text {relman }}(\mathrm{Q}, \mathrm{C})$ is the Manhattan distance between the query and the concept in the data source. $\alpha$ and $\beta$ values ranges from 0 to $1 . \alpha$ and $\beta$ values are to be determined experimentally. For the sample cases $\alpha$ and $\beta$ values used are 0.8 and 0.2 respectively to yield better results.

$\operatorname{Semdist}($ rel $)=\alpha * D_{\text {relstd }}($ relq $)+\beta * D_{\text {relman }}(Q, C)$

(6)

The semantic distance of the geo-spatial concept Semdist(concept) is calculated using equation (7) which is the Manhattan distance.

\section{Semdist $($ concept $)=\mid \operatorname{Semdist}(\operatorname{dim})-\operatorname{Semdist}($ rel $) \mid$}

$\mathrm{D}_{\text {dimstd }}$ returns 0 if query concept dimension, dimq and dimc, concept in data source dimension are equivalent and a value is not equal to 0 if they are not equivalent (the accurate value of $\mathrm{D}_{\text {dimstd }}$ depends on the standardization). The precision and recall values are calculated using equation (8) and equation (9). precision $=\frac{\mid\{\text { relevant concepts }\} \cap\{\text { retrieved concepts }\} \mid}{\mid\{\text { retrieved concepts }\} \mid}$

recall $=\frac{\mid\{\text { relevant concepts }\} \cap\{\text { retrieved concepts }\} \mid}{\mid\{\text { relevant concepts }\} \mid}$

Example

Consider the sample tree shown in Fig.5, the query concept "flooding area" which is defined as "relatively flat area of low-lying land next to a river which is subject to frequent flooding". The ontology mapping is done for the concepts in data source and the concepts are extracted (here "floodplain" is similar and so it will be extracted). The shared vocabulary is used for converting the textual definition into quantitative data (e.g.10 to 60 days waterlogged per year).

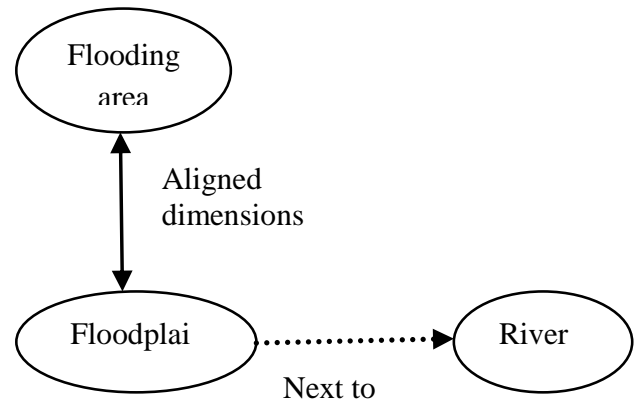

Fig 5: Sample Tree

The data source concept Floodplain is defined by "The relatively flat part of the valley bodering a river resulting from alluvium deposited layer river in times of flood. An area, lowlying, flat-gradient". Consider 2 dimensions such as flat gradient and smooth or even altitude difference is aligned between the geo-spatial query concept "flooding area" and the related concept in datasource "floodplain". Shared vocabulary provides the quantitative data such as $2 \%$ flat gradient and 4 meters of altitude difference for the query concept "flooding area". The quantitative values for the concept in data source "floodplain" are $4 \%$ relatively flat gradient and 5 meters of altitude difference. Assume $\alpha=0.8$ and $\beta=0.2$

$\operatorname{Semdist}(\operatorname{dim})=0.8 * 0+0.2(|4-2|+|5-4|)=0.6$

The concept that is related to flood plain is "river" for which 2 dimensions are aligned such as gradient and altitude difference. The shared vocabulary provides the quantitative data such as $4 \%$ flat gradient and 5 meters of altitude difference for the concept "flood plain". The quantitative values for the concept in data source "river" are $8 \%$ flat gradient and 10 meters of altitude difference.

Semdist $($ rel $)=0.8 * 0+0.2(|8-4|+|10-5|)=1.8$

Semdist $($ concept $)=|0.6-1.8|=1.2$

$\mathrm{D}_{\text {dimstd }}$ returns 0 if query concept dimension ,dimq and dimc, concept in data source dimension are equivalent and a value is not equal 0 if they are not equivalent (the accurate Value of $\mathrm{D}_{\text {dimstd }}$ depends on the standardization). 
This is the way the semantic distance is calculated for the various Geo-spatial concepts. The Geo-spatial concept "Flood plain" having dimensions such as flat gradient and altitude difference as mentioned above when using the Euclidean distance method yields the semantic distance of 6.6 which is more than 2.4 obtained by using Manhattan distance (yields better result). When the semantic distance is more, the similarity is less and vice versa. The Manhattan distance is used for retrieving more similar concepts and computationally easy when compared with the Euclidean distance method.
Further the weights for ontology's geo-spatial concepts are allocated based on the dependency of the context $\mathrm{CD}$ for a given geo-spatial concept C [18]. The weighted ontology [19] is acquired, where heavier sub trees are more context dependent and lighter sub trees are less context dependent. From this the semantic similarity among the geo-spatial concepts can be calculated by taking into account the actual meanings. The positions of the locations [20] are identified by using map based similarity. The next section deals with the conclusion and the future enhancements.

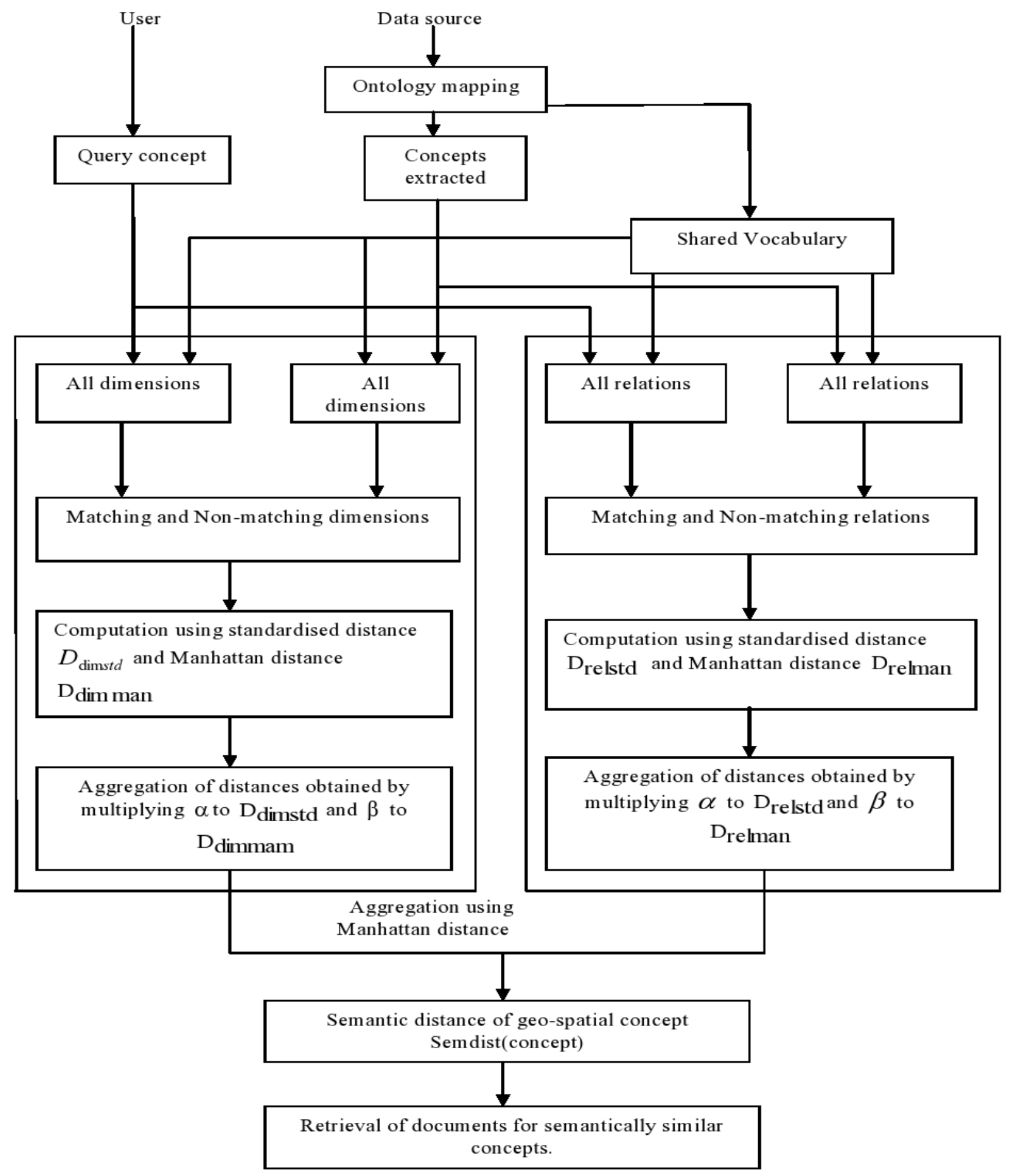

Fig 4: Proposed Hybrid semantic similarity model using Manhattan Distance 


\section{CONCLUSION}

In this study, various computational models for estimating semantic similarity between the geo-spatial concepts are discussed. The survey provides an overview on the drawbacks of the various computational models and depicts how well the hybrid model works. In order to improve the information retrieval, the Hybrid model which uses both the properties and relations to describe the geo-spatial concept is used. The Euclidean distance is used for measuring the semantic similarity between geo-spatial concepts which leads to more number of computations. So the latest Manhattan distance method has been proposed for easier and efficient computation and also it provides better precision and recall for tested geo-spatial concepts. Experiments are to be conducted with different sources having different kinds of spatial relations. The context based similarity is achieved with the assignment of weight to the ontology according to the context dependency for the given geo-spatial concept. And the evaluation other semantic similarity distance measures such as Hirst and orge measure, Sussna distance measure and $\mathrm{Wu}$ palmer distance measure could be done to depict which method provides the better precision and recall.

\section{REFERENCES}

[1] Kay Khaing Win University of ComputerStudies,Yangon, Myanmar, "Measuring Geospatial Semantic Similarity between Geospatial Entity Classes" 0-7803-9521-2/06/\$20.00 \$2006 IEEE.

[2] Schwering, A. (2005). Hybrid model for semantic similarity measurement. 4th International Conference on Ontologies, DataBases, and Applications of Semantics (ODBASE05), Agia Napa, Cyprus, Springer, 1449-1465.

[3] Ahlqvist, O. (2005), "Using Semantic Similarity Metrics to Uncover Category and Land Cover Change". 1st International Conference on GeoSpatial Semantics, Mexico City, Mexico, Springer, 107-119.

[4] Schwering, A. and M. Raubal (2005a), "Measuring semantic similarity between geospatial conceptual regions". $1^{\text {st }}$ International Conference on GeoSpatial Semantics, Mexico City, Mexico, Springer, 93-109.

[5] Goldstone, R. L. and A. Kersten (2003), "Concepts and categorization Comprehensive Handbook of Psychology". A. F. Healy and R. W.Proctor. New Jersey, Wiley. Vol. 4: 599-621

[6] Gärdenfors, P. (2004). How to make the Semantic Web more semantic. Formal Ontology in Information Systems, Proceedings of the Third International Conference (FOIS2004). L. Vieu. Amsterdam, NL, IOS Press. 114: 153-164.

[7] Goldstone, R. L. (1994b). Similarity, Interactive Activation, and Mapping. Journal of Experimental Psychology: Learning, Memory, and Cognition 20(1): 328.

[8] Tversky, B. and P. U. Lee (1998). How space structures language. Spatial cognition: An interdisciplinary approach to representation and processing of spatial knowledge, Springer, 157-175.
[9] Rodríguez, A. and M. J. Egenhofer (2003). Determining semantic similarity among entity classes from different ontologies. IEEE Transactions on Knowledge and Data Engineering 15(2): 442-456.

[10] Li, B. and F. T. Fonseca (2006), "TDD - A Comprehensive Model for Qualitative Spatial Similarity Assessment." Spatial Cognition and Computation 6(1): 31-62. pre print version

[11] Schwering, A. and M. Raubal (2005b). Spatial relations for semantic similarity measurement. 2nd International Workshop on Conceptual Modeling for Geographic Information Systems (CoMoGIS2005), Klagenfurt, Austria., Springer, 259-269.

[12] A. Vadivel, A.K. Majumdar,Shamik Sural “ performance comparison of distance metrics in content-based Image retrieval applications" Computer Science and Engineering, Indian Institute of Technology, Kharagpur,

[13] Sanjay Chawla, Shashi Shekhar, Weili Wu, and Uygar Ozesmi "Modeling Spatial Dependencies for Mining Geospatial Data"

[14] Rodríguez, A. and M. J. Egenhofer (2004). Comparing geospatial entity classes: An asymmetric and contextdependent similarity measure. International Journal of Geographical Information Science 18(3): 229-256.

[15] Resnik, P. (1999). Semantic similarity in a taxonomy: An information-based measure and its application to problems of ambiguity in natural language. Journal of Artificial Intelligence Research 11: 95-130.

[16] Levenshtein, I. V. (1966). Binary codes capable of correcting deletions, insertions, and reversals. Soviet Physics Doklady 10(8): 707-710.

[17] Hosseinabady, M. and Nunez-Yanez, "Run-time stochastic task mapping on a large scale network-on-chip with dynamically reconfigurable tiles", J.L. Computers \& Digital Techniques, IET Volume: 6, Issue: 1 Digital Object Identifier: 10.1049/iet-cdt.2010.0097 Publication Year: 2012 , Page(s): 1 - 11 IET Journals \& Magazines

[18] Ahmad El Sayed, Hakim Hacid, Djamel Zighed, "Estimating Semantic Distance Between Concepts for Semantic Heterogeneous Information Retrieval" University of Lyon 2 ERIC Laboratory- 5, avenue Pierre Mend`es-France 69676

[19] Jay J. Jiang David W. Conrath, "Semantic Similarity Based on Corpus Statistics and Lexical Taxonomy" Department of Management Sciences MGD School of Business University of Waterloo McMaster University ,Ontario, Canada N2L 3G1Hamilton, Ontario, Canada

[20] Montello, D. R., S. I. Fabrikant, M. Ruocco and R. S. Middleton (2003). Testing the first law of cognitive geography on point-display spatializations. Conference on Spatial Information Theory (COSIT03), Kartause Ittingen, Switzerland, Springer, 316-331. 